\section{Analysis of Fire Disasters and Burns in Taiwan Lee-Min Wang, MD; ${ }^{1}$ S.C. Hu, MD; ${ }^{1}$ C.H. Lee, $M D ;^{2} J . R$. Chiang ${ }^{1}$}

1. Emergency Department,Veterans General Hospital, Taipei Society of Emergency and Critical Care Medicine, Taipei, Taiwan 2. National Fire Administration, Ministry of the Interior, Taiwan, Republic of China

Disasters are tragedies that overwhelm our communities, destroy our property, and harm our populations. In recent years, because the environment has been changing from agriculture to industry, the fire disaster has become the major cause of damage to people's life and property in our society.

Fire disaster differs from any other form of disaster in a number of specific ways. It differs first of all in its cause, fire particularly has an increased destructive effect on material goods and livings. For preventive purposes, we report on an analysis of fire disasters in Taiwan from 1978 to 19.96 including:

1) Incidence - the two peak years were 1983 and 1986

2) Season index - the most common months are January, October, November and December;

3) Cause-except unknown origin, electrical, wilderness fires, smoking, cooking, and mechanical abrasion;

4) Duration-first: from $12: 00$ to $15: 00$, second: from 15:00 to $18: 00$;

5) Mortality-the mortality depends on the increase in population using the city rate: Taiwan, $2.77 \%$; Taipei, 6.18\%; Kaohsiung:,4.42\%;

6) Burns - by age and gender: peak in 0-2 years and 19-45 years; Male > Female; and

7) Causes of burns - most frequent relate to scalding injuries, second: flame.

Conclusion: Fire disasters occur during the Winter season and in the afternoon; the causes of fire disaster depend on building structure; and the mortality rate of fire disaster is high in the city. Therefore, prevention of fire disasters is important:

1) Efficiency of fire defense system in buildings is directly or indirectly involved;

2) Efficient program of education of the population must be popular and must include the public and the operative, medical, paramedical, and voluntary forces;

3) Development of plans in the medical hospital so that they are prepared against fire disasters;

4) Establishment of the triage pathway for burned patients in fire disasters; and

5) Setting up a network of prehospital care, emergency medical services system and in-hospital care during fire disasters.

Key Words: disaster; fire; fire disaster; incidence; mortality; prevention; seasons; Taiwan
The Earthquake in the Aegion Region in 1995

N. Zachopulos; ${ }^{1}$ E. Kontospyru, ${ }^{1}$ G. Letsios; ${ }^{1}$

S. Bakas, ${ }^{1}$ D. Lambropulos; ${ }^{1}$ V. Stergiopulos, ${ }^{1}$

M. Kokoliu; ${ }^{1}$ C. Bachtis; ${ }^{2}$ M. Vaphiadu ${ }^{2}$

1. Nursing Office of the HNEMS, 2.Medical Department of the HNEMS, Hellenic National Emergency Medical Service, Athens, Greece

On 15 June, 1995 at 03:15 h, an earthquake of 6.1 on the Richter scale, struck the major area of the city of Aegio in Peloponissos, approximately $180 \mathrm{~km}$ southwest of Athens. Only one hour after the notification of the Special Branch of Disaster Medicine of the Hellenic National Emergency Medical Service (HNEMS), the first rescue mission comprised of two Mobile Medical Units (MMU) and an ambulance (A) manned with emergency physicians, nurses and rescuers, and carrying special rescue equipment was dispatched to the scene. A further group of two emergency physicians and a disaster manager were brought by helicopter to the city of Aegio.

After the first evaluation of the situation on site, the needs and priorities were established and two rescue groups were dispatched, one to a hotel at Valimitika and one to an apartment house in Aegio city. They began the rescue.

Within hours, more rescue groups arrived from Athens, Patras, and Thessaloniki, as well as from France and Switzerland. Twenty people were extricated dead. Fifteen needed immediate treatment and nursing care.

Conclusion: The rapid deployment of the Special Branch of Disaster Medicine of the HNEMS at the disaster site was of crucial importance. With the cooperation of the other rescue groups, they saved lives and relieved the earthquake victims.

Key Words: disaster; earthquake; rescue; response; response time

\section{The Emergency Care \\ in the China Tangshan Earthquake}

Li Zong Hao, $M D$

Vice President of Chinese Association of Emergency Medicine, Medical Director of Beijing Emergency Medical Center

In the early morning on 28 July 1978, there happened an earthquake in Tangshan City in China that measured 7.8 on Richter Scale. Some 0.95 million people were injured, and among which 0.243 million people died. Several thousand people were permanently disabled.

The earthquake extended to Beijing and Tianjin. The author worked as the chief duty director of the BEMC at the time, and soon after, worked in area where the injured people gathered and provided the on-site emergency care at the transportation hub.

The author suggested that Rescue Emergency Medicine should be founded in the discipline of Emergency Medicine (EM) and Disaster Medicine (DM) so as to satisfy the requirement of the on-site emergency care and medical caring for victims of disasters in modern society. Medical care in severe circumstances cannot operate 
simply using regular medical skills.

The author suggests that international colleagues support each other in large disasters and develop academic exchanges so as to improve emergency care organization and skills.

Key Words: deaths; disabilities; earthquake; injuries; Rescue Emergency Medicine

\section{A Nuclear Disaster Handling Manual}

Y. Haragucbi; H. Nishi; Y. Tomoyasu; T. Arai

Clinical Research Institute, National Hospital Tokyo

Disaster Medical Center, Tachikawa City, Tokyo, Japan

This March 1997, a large scale of accident in a nuclear waste plant occurred in Tokaimura, which is located at a distance of approximately $150 \mathrm{~km}$ from the center of Tokyo. More than 30 people who were working in the plant were contaminated. This accident reminds us of the potential for the occurrence of mass casualties by radioactive substances.

In the event of a disaster, the National Hospital Tokyo Disaster Medical Center will take charge of disaster-related medical care. In this Institute, we have been developing a disaster manual that focuses on the handling of casualties subjected to nuclear contamination. The development of this manual is discussed.

At the present, many problems remain when treating casualties contaminated with radioactive substances. Among them was the difficulty of determining the existence of the contamination with accuracy, effective methods for decontamination of the victims, the use of drugs that will promote excretion of toxic substances, transportation of patients, special rooms where contaminated patients are admitted, the storage and disposal of the polluted materials and water, and legal problems

Key Words: contamination; manual decontamination; nuclear disaster; radioactive substances

\section{Analysis of the Kinds of Diseases Encountered in the Shanghai Prehospital Emergency Care Service: 1991-1995 \\ $X u$ Huiliang \\ National Training Center for Emergency Care Personnel, Shanghai, Peoples Republic of China,}

The author analyzed 467,674 cases of prehospital emergency care provided by the Shanghai Medical First-Aid Center during 1991-1995. The aim of this analysis was to determine the disease spectrum of prehospital emergency care provided in Shanghai, and to identify ways by which to improve the level of prehospital emergency care.

Results: There were 268,300 males cases $(57.3 \%)$ and 199,374 female cases $(42.6 \%)$. The ratio of male to female was $1: 345.49 .2 \%$ of the patients were $\geq 60$ years of age; $20 \%$ of the cases were critically diseased or injured. The five leading causes that demanded prehospital emergency care in rank order were: 1) traumatic injuries; 2) cerebrovascular diseases; 3) cardiac diseases; 4) acute abdominal pain; and 5) respiratory diseases. The five leading causes of prehospital deaths were: 1) cardiac diseases; 2) suicides and intoxication; 3) respiratory emergencies; 4) cerebrovascular diseases; and 5) neoplasms. The results also showed the characteristics of monthly distribution of several disorders.

Conclusion: Cardiac diseases, cerebrovascular diseases, respiratory tract diseases, injuries, and intoxication should be the focal points for prehospital emergency care instruction, and ambulance equipment and drug supplies should fit the demands of these diseases and fit the seasonal changes in disease spectrum.

Key Words: causes of death; disease distribution; prehospital emergency care; Shanghai

\section{The Disastrous Epidemic 0-157}

(Escherichia Coli) Food Poisoning in Osaka

Arito Kaji; Tosbinori Miyaicbi; Hirosbi Rinka;

Yoshio Matsuo; Masanori Kan; Tatsubiro Shigemoto;

Takahisa Yosbimura; Kazuma Tsukioka; Takashi Ukai

Emergency and Critical Care Medical Center, Osaka

City General Hospital, Osaka, Japan

In July 1996, more than 6,000 children suffered from acute food poising caused by Enterohemorrhagic Escherichia Coli-0-157 after eating school lunches in Sakai City of the Osaka Prefecture. Sakai City has a population of approximately 900,000 and neighbors Osaka City (about $20 \mathrm{~km}$ to the south).

Severe diarrhea, vomiting, and bloody stools led some children to consult local physicians on 11 July, but the number of victims increased abruptly on 13 July (Saturday), and overwhelmed the capacity of pediatric medical services in Sakai. The number of children who needed admission was 32 on $13 \mathrm{July}$, and increased to 493 on the next day. Hospitals in Sakai were so crowded with the ill children that some of the children had to wait 6 hours for consultation. On the other hand, hospitals in Osaka City (neighboring city to Sakai) were not crowded, and the out-patient departments and wards were quiet as in a normal weekend.

The Osaka Prefecture Emergency Medical Information Center (OPEMIC) looked for vacant beds in all of the Osaka Prefecture to Sakai Fire Department and Health Bureau of Sakai City Government from the evening of 13 July; 152 patients were transferred to 44 hospitals in other cities. Osaka City General Hospital (OCGH) received 15 moderately ill patients in the first two days. As all the pediatric beds of OCGH were occupied on the afternoon of 13 July, six additional beds were prepared in the emergency center. Eight more patients were transferred to OCGH by the request of OPEMIC. In total, 23 children were admitted and six of them were seriously ill due to dehydration and/or acute renal failure (hemolytic uremic syndrome).

All of the patients recovered within one month without any sequellae. However, three children treated in other hospitals died from Hemolytic Uremic Syndrome and encephalopathy.

This disastrous epidemic was inadequately recognized. The congestion and the confusion of the hospitals in Sakai in the first few days could have been avoided. 